\title{
The nonstructural proteins of Pneumoviruses are remarkably distinct in substrate diversity and specificity
}

\author{
Michael Ribaudo ${ }^{1}$ and Sailen Barik ${ }^{1,2^{*}}$
}

\begin{abstract}
Background: Interferon (IFN) inhibits viruses by inducing several hundred cellular genes, aptly named 'interferon (IFN)-stimulated genes' (ISGs). The only two RNA viruses of the Pneumovirus genus of the Paramyxoviridae family, namely Respiratory Syncytial Virus (RSV) and Pneumonia Virus of Mice (PVM), each encode two nonstructural (NS) proteins that share no sequence similarity but yet suppress IFN. Since suppression of IFN underlies the ability of these viruses to replicate in the host cells, the mechanism of such suppression has become an important area of research. This Short Report is an important extension of our previous efforts in defining this mechanism.

Results: We show that, like their PVM counterparts, the RSV NS proteins also target multiple members of the ISG family. While significantly extending the substrate repertoire of the RSV NS proteins, these results, unexpectedly, also reveal that the target preferences of the NS proteins of the two viruses are entirely different. This is surprising since the two Pneumoviruses are phylogenetically close with similar genome organization and gene function, and the NS proteins of both also serve as suppressors of host IFN response.
\end{abstract}

Conclusion: The finding that the NS proteins of the two highly similar viruses suppress entirely different members of the ISG family raises intriguing questions of pneumoviral NS evolution and mechanism of action.

Keywords: Paramyxovirus, Pneumovirus, Respiratory syncytial virus, Interferon, ISG

\section{Introduction}

IFN is recognized as an antiviral cytokine that uses STAT-family transcription factors to induce a few hundred IFN-stimulated genes (ISGs), many of which have been shown to be antiviral $[1,2]$. Several RNA viruses of the Paramyxoviridae family, however, exhibit IFN resistance $[3,4]$. We have been following this property in selected members of this family [5-9], focusing on the Pneumovirus genus, which is comprised of two members that are severe respiratory pathogens, namely RSV, a human virus, and PVM, a mouse virus [10, 11]. A natural mouse pathogen, PVM is lethal in laboratory mice, producing symptoms that are very similar to those of RSV disease in susceptible human subjects, whereas RSV itself replicates relatively poorly in mice, without

\footnotetext{
*Correspondence: barikfamily@gmail.com

'Department of Biological, Geological and Environmental Sciences, and Center for Gene Regulation in Health and Disease, Cleveland State University, 2121 Euclid Avenue, Cleveland, OH 44115, USA

23780 Pelham Drive, Mobile, AL 36619, USA
}

significant mortality [10-12]. Thus, PVM infection in mice has often been recommended as an alternative model for human RSV infection [12]. Regarding innate immunity of the host, both viruses code for two nonstructural proteins, NS1 and NS2, that suppress IFN, which is essential for robust growth of the virus and the resultant pathology [13-23]. However, despite intensive research, the exact molecular mechanism of this important function of NS proteins remains unknown [6-8, 24-27]. Intriguingly, the primary structures of the NS proteins show no similarity with any other protein in biology; thus, bioinformatic analysis of their sequences offers no clue to their evolutionary origin or functional domains $[6,9,24]$. Except for the recently solved crystal structure of RSV NS1 [28], the higher order structure of the pneumoviral NS proteins has also remained unknown, in part due to difficulties of expression of the recombinant proteins; moreover, the crystal structure of RSV NS1 [28] did not offer any mechanistic insight into its IFN-suppressive function. Nonetheless, we have recently shown that the 
PVM NS proteins specifically degrade several mouse ISGs, namely IFITM1, TRAFD1 and ISG20 [24]. At this point, we argued that a comparison between the NS proteins of the two pneumoviruses may shed light on their structure and function. Thus, we proceeded to test if the RSV NS proteins target any ISGs, and if so, whether they are same or different from those targeted by their PVM counterparts.

\section{Methods}

Recombinant NS clones, cell culture, transfection and immunoblot assays have been published [6, 7, 24, 29, 30] and the overall experiment followed our optimized procedure described previously [24]. In brief, codonoptimized NS1 and NS2 cDNA sequences were cloned in pCAGGS plasmid such that the proteins are expressed with FLAG tag at the amino terminus [6]. Construction of the HEK293 panel of tetracycline (Tet)inducible FLAG-tagged ISG cells and their culture conditions have been described in detail [24, 29, 30]. The cells were grown in monolayer in Dulbecco's minimum essential media (D-MEM), supplemented with Lglutamine, tetracycline-free fetal bovine serum (Omega Scientific, Tarzana, CA; FB-15/100; 10\%), penicillin $(100 \mathrm{IU} / \mathrm{ml})$, streptomycin $(100 \mu \mathrm{g} / \mathrm{ml})$, hygromycin $(250 \mu \mathrm{g} / \mathrm{ml})$ and blasticidin $(5 \mu \mathrm{g} / \mathrm{ml})$. At near confluency, the cells were transfected with $1.6 \mu \mathrm{g}$ FLAG-NS1 or $0.8 \mu \mathrm{g}$ FLAG-NS2 plasmid or both $(0.8 \mu \mathrm{g} / 0.8 \mu \mathrm{g})$, using
Lipofectamine 2000 (Invitrogen/Life Technologies) following the manufacturer's protocol. The FLAG-ISG proteins were induced with Tet $(1 \mu \mathrm{g} / \mathrm{ml}) 24 \mathrm{~h}$ after transfection, and after another $24 \mathrm{~h}$, cells were harvested for immunoblot analysis. Total cell extracts were made, and the indicated FLAG-ISG and FLAG-NS proteins were detected using FLAG antibody (Sigma, SLBF6631/F1804) as primary antibody. GAPDH served as the loading control, detected by primary mouse antibody (Santa Cruz, sc365,062). The HRP-conjugated secondary antibody was anti-mouse, raised in goat (Santa Cruz, sc-2031). HRP was developed by ECL reaction using Prime Western Blotting Detection Reagent (GE Healthcare) and detected in the LI-COR Odyssey Fc imaging system. The band intensities in the immunoblot images were quantified and analyzed by NIH ImageJ software, and graphs were drawn by Excel using average values and standard deviations. Multiple sequence alignment and the resultant cladogram were obtained by using Clustal Omega [31].

\section{Results}

To start with, we examined the effect of RSV NS proteins on six major human ISGs, and the immunoblot results are shown (Fig. 1). As described previously, the original tet-inducible ISG cell panel contained 25 different ISGs that are abundantly induced by IFN [29, 30]; from these, we chose to test six because of the better growth of the cells and robust Tet-inducibility. Out of

\section{a}
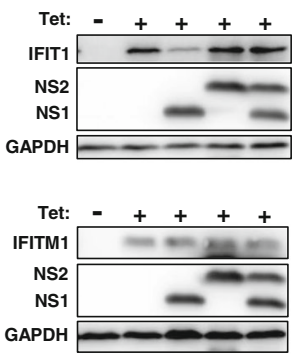

b

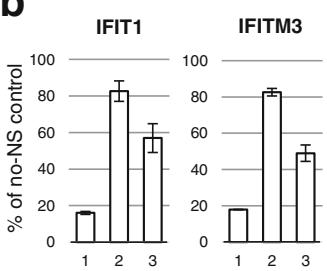

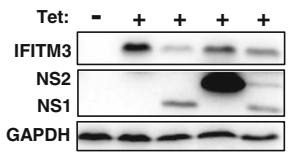
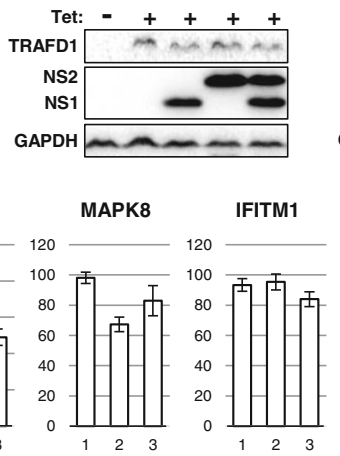
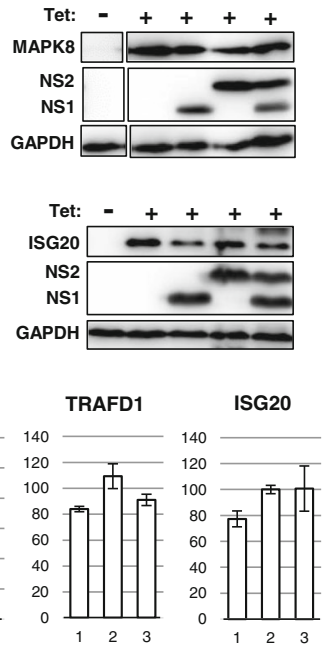

Fig. 1 Reduction of the steady-state levels of specific ISGs by RSV NS proteins. These experiments are detailed in Methods, and representative results are shown. Briefly, Tet-inducible, stably transfected FLAG-tagged ISG cells were transiently transfected with the indicated FLAG-NS1 and -NS2 plasmids, as shown. The cells were induced with Tet (Tet +), while control cultures remained uninduced (Tet -). Immunoblotting was performed to quantify the FLAG-NS proteins and residual FLAG-ISG proteins. The immunoblot (panel a), and a bar graph of the ISG band intensities on the blot, expressed as percent of the 'no-NS' amount (panel b), are shown. GAPDH served as internal control, to which the ISG intensities were normalized for the plot. In panel $\mathbf{b}$, for all ISGS, bar 1 means NS1 only, bar 2 means NS2 only, bar 3 means both NS1 and NS2 were transfected. Each bar is average of three measurements with the standard deviation shown 
the six ISGs tested, the RSV NS proteins reduced the steady-state levels of three, namely IFIT1, IFITM3, and MAPK8 (Fig. 1), although NS1 and NS2 showed preferences, as clearly seen in Table 1.

Whereas IFIT1 and IFITM3 were preferentially targeted by RSV NS1, MAPK8 was a preferred target of NS2. The degree of reduction was also different such that NS1 lowered the steady-state levels of IFIT1 and IFITM3 by greater than $80 \%$, but NS2 reduced MAPK 8 levels only by a third. To our surprise, however, they had little or no effect on the human counterparts of the PVM NS substrates, i.e. IFITM1, TRAFD1 and ISG20. Whereas TRAFD1 and ISG20 showed a slight sensitivity to RSV NS1 (16\% and 23\% reduction, respectively), they were essentially fully refractory to RSV NS2. It is to note that the PVM NS proteins also preferred as well as shared their targets to various degrees. Regardless, it seems fair to assume that screening of the full set of $>400$ ISGs [2] may lead to the identification of many more differential NS targets of PVM and RSV. We conclude that the full range of ISGs targeted by the pneumoviral NS proteins may be much broader than previously appreciated.

Given the differential substrate choice of the NS proteins of RSV and PVM, we wondered whether NS protein sequences had any common motif or domain that might shed light on their common function as IFN antagonists. To this end, we compared the conceptually translated amino acid sequences of the NS1 and NS2 proteins of RSV and PVM, in order to find even short regions of homology. However, as shown (Fig. 2), no major sequence similarity could be discerned among all four of them. This dissimilarity of NS proteins contrasts the significant sequence similarity between the other proteins of the two viruses, which are structural proteins, such as the $\mathrm{N}$ (nucleocapsid) proteins sharing 61\% amino acid identity, the $\mathrm{P}$ (phosphoprotein) proteins sharing 33\% identity, and the $M$ (matrix) proteins sharing $43 \%$ identity [11]. Nevertheless, the NS2 proteins of the two viruses deserve special mention. Previous studies from our group and others $[5-7,26]$ showed that NS2 of human RSV can degrade the host STAT2 protein, likely by recruiting the ubiquitin-mediated proteasome system. Very recently, extensive deletion and sitedirected alanine mutagenesis of RSV NS2 protein led to the interesting finding that multiple amino acids spanning the entire length of NS2 are required for optimal ubiquitination of host proteins [32]. Five residues were identified as playing the most important role in this function (Fig. 2); in RSV NS2 sequence, they were T36, L52, P92, and C105, among which T36 was the strongest contributor [32]. As noted earlier, these residues are also conserved in the PVM NS2 sequence in the alignment (Fig. 2), the significance of which is currently unknown, since the ubiquitination function of PVM NS2 has not been investigated in depth $[24,32]$. Taken together, these observations underscore diversity as well as specificity of target selection by the biologically unique pneumoviral nonstructural proteins.

\section{Discussion}

The distinctive substrate repertoire of the two pneumoviral NS proteins agrees with their widely different primary structures, indicating that the NS proteins may have different mechanisms of action that need to be unraveled. As mentioned, there is very limited conservation of key residues, such as T36, in the NS2 proteins of the two viruses, whose significance is unknown [32]. Recently determined crystal structure of RSV NS1 showed some similarity of structural fold with the RSV M protein [28]. The significance of this exclusive similarity at the higher structural level is also enigmatic since the $\mathrm{M}$ protein is a virion structural protein that links the genome RNA nucleocapsid to the viral envelope, with no known role in IFN suppression. RSV NS1 promotes ubiquitin-dependent proteasomal degradation of human STAT2 [27], and in PVM, both NS1 and NS2 also have similar degradative effect on mouse STAT2 [24]. However, the lack of any sequence similarity among these proteins currently does not allow any insight into their

Table 1 Contrasting activity of RSV and PVM nonstructural proteins on select ISGS

\begin{tabular}{llll}
\hline ISG name & GenBank\# & \% Reduction by RSV NS1 or NS2 & \% Reduction by PVM NS1 or NS2 [24] \\
\hline IFIT1 & NM_001548 & $84 \%$ NS1, 17\% NS2 & Not done \\
IFITM3 & NM_021034 & $82 \%$ NS1, 17\% NS2 & Not done \\
MAPK8 & AB451231.1 & $2 \%$ NS1, 33\% NS2 & Not done \\
IFITM1 & NM_003641 & $7 \%$ NS1, 5\% NS2 & $63 \%$ NS1, 32\% NS2 \\
TRAFD1 & NM_006700 & $16 \%$ NS1, 0\% NS2 & $68 \%$ NS1, 76\% NS2 \\
ISG20 & NM_002201 & $23 \%$ NS1, 0\% NS2 & $18 \%$ NS1, 54\% NS2
\end{tabular}

This is a numerical summary of reduction of the selected ISGs by the two pneumoviral NS proteins, expressed individually. The RSV data were calculated with the average band intensities of the immunoblot of Fig. 1a, which were also graphically plotted in Fig. 1b, and the PVM data were calculated by densitometry of the corresponding immunoblot in our recent publication [24]. "Not done" indicates ISGs for which recombinant mouse homologs were unavailable. Note that in this Table we present the percent reduction of the ISG, whereas in Fig. 1b (bar graph), the percent remaining is plotted; thus $84 \%$ reduction of ISG here corresponds to $16 \%$ remaining ISG in Fig. 1b. RSV NS proteins affected the upper three ISGs are more strongly than the lower three 


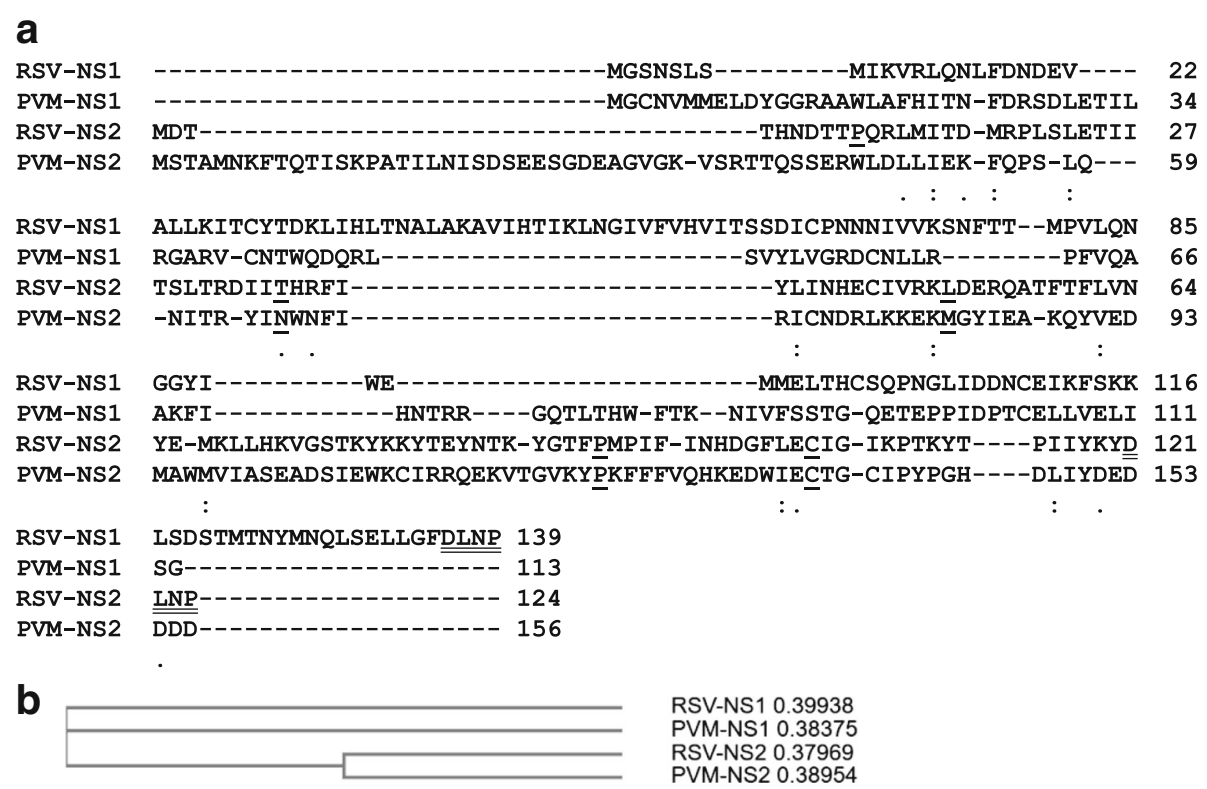

Fig. 2 Multiple sequence alignment of the RSV and PVM NS proteins. The GenBank accession numbers are: NP_044589.1 (RSV NS1), AHW80505.1 (RSV NS2), YP_173324.1 (PVM NS1), YP_173325.1 (PVM NS2). a Alignment by Clustal Omega, essentially showing the lack of any significant similarity among these proteins, perhaps in agreement with their differential effect on various ISGs. Only a few conservative replacements are denoted by dots. Amino acid residues, mapped to be important for RSV NS2 ubiquitination function [32], are underlined; note that they are also conserved in PVM NS2, in spite of the high dissimilarity in the overall sequences. The C-terminal tetrapeptide DLNP, double-underlined in the two NS proteins of RSV, may have a functional role in substrate degradation, as shown before [7]; it is not found in the PVM NS sequences. b Standard cladogram with similarity scores, generated by the default parameters of the same program, reveals that the NS1 and NS2 orthologs of the two viruses are slightly more similar to each other than NS1 and NS2 of the same virus, perhaps suggesting that NS1 and NS2 evolved independently

mechanism. This is in contrast, for example, the IFNantagonist $\mathrm{V}$ proteins of the Rubulavirus genus of this family, all of which degrade STAT proteins by assembling cellular proteasomes and possess conserved sequences such as a Cys-rich zinc-binding domain [33]. Although there is experimental evidence that RSV NS proteins recruit ubiquitin-dependent proteasomal complexes, the exact composition and substrate specificity of these complexes remain unknown; it is also unknown whether PVM NS proteins form similar complexes $[6-9,24,27]$. It is unlikely that there will be a common sequence motif among all the ISG substrates that the NS proteins recognize. At the same time, the NS proteins do not affect the vast majority of cellular proteins including the NS-resistant ISGs. Of note, both NS1 and NS2 of RSV have been shown to possess several other functions that need to be reconciled with their ISG-targeting activity; such functions include inhibition of apoptosis by both proteins [34,35], regulation of cell cycle by NS1, NS2-mediated direct inhibition of RIG-I (retinoic acid-inducible gene I) [36], which is the proximal sensor of viral RNA in the IFN induction cascade, and NS1-mediated reduction of the transcriptional activity of IRF3 (IFN regulatory factor 3), which is a transcription factor for the IFN genes [37]. Clearly, the molecular mechanism of how the sequence diversity of the pneumoviral NS proteins as well as their multiple substrates still allow target specificity will be a fascinating area of future research.

At this time, we have not screened the ISGs for their ability to inhibit RSV; however, all three NS substrates reported here have a strong likelihood to function as antiviral. The IFIT (interferon-induced proteins with tetratricopeptide repeats) family in human consists of four TPR-domain proteins, of which IFIT1 is the most abundantly induced, following exposure of the cells to IFN [38]. Recent studies have revealed strong antiviral activity of IFIT1 against several viruses [38-46], including two viruses of the Paramyxoviridae family, i.e. PIV3 and PIV5 $[29,41]$. IFIT1 inhibits the translation of mRNA with unmethylated 5' -cap [42-46] and RSV mRNA caps may be only partially methylated $[47,48]$. Thus, RSV may be significantly sensitive to IFIT1-mediated translational inhibition. As proposed previously, IFIT1 may also recognize 5 '-triphosphate (5'-ppp) viral RNA [49], such as the RSV leader RNA or leader-readthrough RNA $[50,51]$, but the outcome of these interactions cannot be easily predicted.

IFITM3, a member of the IFITM (IFN-induced transmembrane protein) family of proteins, is located in the endocytic membrane; it has been shown to strongly inhibit West Nile virus (WNV) and dengue virus (DENV) 
[52], but had no effect on PIV3 [29], likely because fusion of paramyxoviruses including PIV3 occurs at the cell surface without an endocytic requirement. RSV, however, is an exception in this family, as it depends on endocytic entry for the activation of its fusion protein (F) by a second proteolytic cleavage [53]. It is thus conceivable that endocytic IFITM3 may act as a roadblock to RSV entry and processing.

In contrast, IFITM1 is the only member of the IFITM family that is localized in the cell membrane [54, 55]; it is not a target of RSV NS proteins (Fig. 1) and may also have no role in RSV entry or egress. Lastly, the role of MAPK8 (Mitogen Activated Protein Kinase 8) in viral regulation may take many forms, since MAP kinases in general act as an integration point for myriad cellular signals [56], some of which may affect RSV. We do note that the ability of NS to target a host innate immune protein may not necessarily correlate with the ability of the protein to inhibit the virus, even though it makes evolutionary sense from the viral standpoint; in other words, we cannot conceive of a simple protein-protein interaction mechanism by which the NS proteins will exclusively recognize those proteins of the host cell that possess antiviral activity. Regardless, targeting the ISGs may have endowed pneumoviruses with superior IFN resistance; for example, one of the earliest comparative studies revealed that IFN concentrations of $10,000 \mathrm{U} / \mathrm{ml}$ inhibits RSV growth in cell culture by only $\sim 15$-fold while reducing PIV3 growth by 1000- to 10,000-fold [57].

\section{Conclusion}

Our results have shown that the IFN-antagonistic pneumoviral nonstructural (NS) proteins, which are essential for optimal virus growth, suppress a much larger repertoire of host IFN-pathway factors than was previously conceived. Acting singly and/or together, the NS1 and NS2 proteins of both pneumoviruses, namely RSV and PVM, reduce the levels of multiple ISG proteins, the ultimate antiviral factors of the IFN system. The highly dissimilar and unique primary structures of the NS proteins and their ability to target equally diverse ISGs, but in a specific manner, have raised intriguing questions about their evolution and mechanisms of action.

\section{Limitations}

We have used ISG over-expressing cell lines here, which may not produce exactly the same amount of ISG proteins as in an IFN-stimulated cell. These cell lines are also in HEK293 background, which are not physiological hosts of RSV infection. However, the RSV NS proteins can suppress a wide range of substrate concentration, expressed from diverse vectors [6, 7, 27], and can also antagonize IFN concentrations varying over a range of 500-10,000 U/ml [57]. Regarding the recombinant expression of NS proteins, we have used single amounts of the pCAGGS-NS plasmids that were pre-optimized to produce the same amounts of NS as in RSV-infected cells [6]. We used HEK293 cells, which are deficient in the STING-cGAS pathway [58, 59], to avoid spurious induction of IFN response by the transfected NS plasmids, since recent studies have discovered that this pathway is activated by cytoplasmic DNA. Finally, we have assumed that the RSV NS proteins promote degradation of the ISGs tested here, based on the proteasomal degradation of several other IFN-pathway proteins by these NS proteins, observed by several groups, as described earlier. However, our attempt to directly test this by the use of proteasomal inhibitors, such as MG132, met with technical problems because it was difficult to find an optimal concentration of MG132 that would significantly restore ISG levels without exerting cytotoxic effect on the HEK293 strain in which the ISG cell lines are created. Evidently, the mechanism of reduction of diverse ISGs by NS proteins will await further studies.

\section{Abbreviations \\ HRP: Horseradish peroxidase; IFIT: Interferon-induced proteins with tetratricopeptide repeats; IFITM: Interferon-induced transmembrane protein; IFN: Interferon; ISG: Interferon-stimulated gene; MAP K8: Mitogen-activated protein kinase 8; NS: Nonstructural; PIV3: Parainfluenza virus type 3; PVM: Pneumonia virus of mice; RSV: Respiratory syncytial virus; STAT: Signal transducers and activators of transcription; Tet: Tetracycline; \\ TPR: Tetratricopeptide repeat; TRAFD1: TRAF-type zinc finger domain- containing protein 1}

Acknowledgements

We thank Dr. Ju-Tao Guo (Baruch S. Blumberg Institute, PA, USA) for the ISG-expressing cell lines.

\section{Funding}

This research was supported in part by NIH grant Al109569. The publication cost of this paper was paid by the personal funds of SB.

\section{Availability of data and materials}

All data generated and analyzed during this study are included in this article. No recombinant plasmid was constructed specifically for this study.

\section{Authors' contributions}

MR contributed to experiments, design, and analysis, and SB to concept, analysis, and writing. Both authors read and approved the manuscript at multiple stages.

\section{Ethics approval}

No animals or human subjects were used in these studies.

Consent for publication

Not applicable.

Competing interests

The authors declare that they have no competing interests.

\section{Publisher's Note}

Springer Nature remains neutral with regard to jurisdictional claims in published maps and institutional affiliations. 
Received: 1 September 2017 Accepted: 27 October 2017 Published online: 06 November 2017

\section{References}

1. Borden EC, Williams BR. Interferon-stimulated genes and their protein products: what and how? J Interf Cytokine Res. 2011;31:1-4

2. Schneider WM, Chevillotte MD, Rice CM. Interferon-stimulated genes: a complex web of host defenses. Annu Rev Immunol. 2014;32:513-45.

3. Chatterjee S, Basler CF, Amarasinghe GK, et al. Molecular mechanisms of innate immune inhibition by non-segmented negative-sense RNA viruses. J Mol Biol. 2016:428:3467-82.

4. Devasthanam AS. Mechanisms underlying the inhibition of interferon signaling by viruses. Virulence. 2014;5:270-7.

5. Ramaswamy M, Shi L, Varga SM, et al. Respiratory syncytial virus nonstructural protein 2 specifically inhibits type I interferon signal transduction. Virology. 2006;344:328-39.

6. Swedan S, Musiyenko A, Barik S. Respiratory syncytial virus nonstructural proteins decrease levels of multiple members of the cellular interferon pathways. J Virol. 2009;83:9682-93.

7. Swedan S, Andrews J, Majumdar T, et al. Multiple functional domains and complexes of the two nonstructural proteins of human respiratory syncytial virus contribute to interferon suppression and cellular location J Virol. 2011;85:10090-100

8. Goswami R, Majumdar T, Dhar J, et al. Viral degradasome hijacks mitochondria to suppress innate immunity. Cell Res. 2013;23:1025-42.

9. Barik S. Respiratory syncytial virus mechanisms to interfere with type 1 interferons. Curr Top Microbiol Immunol. 2013;372:173-91.

10. Horsfall FL, Hahn RG. A pneumonia virus of Swiss mice. Proc Soc Exp Biol Med. 1939;40:684-6.

11. Easton AJ, Domachowske JB, Rosenberg HF. Animal pneumoviruses: molecular genetics and pathogenesis. Clin Microbiol Rev. 2004;17:390-412

12. Rosenberg HF, Domachowske JB. Pneumonia virus of mice: severe respiratory infection in a natural host. Immunol Lett. 2008;118:6-12.

13. Krempl CD, Lamirande EW, Collins PL. Complete sequence of the RNA genome of pneumonia virus of mice (PVM). Virus Genes. 2005;30:237-49.

14. Teng MN, Collins PL. Altered growth characteristics of recombinant respiratory syncytial viruses which do not produce NS2 protein. J Virol. 1999;73:466-73.

15. Teng MN, Whitehead SS, Bermingham A, et al. Recombinant respiratory syncytial virus that does not express the NS1 or M2-2 protein is highly attenuated and immunogenic in chimpanzees. J Virol. 2000;74:9317-21.

16. Bossert B, Marozin S, Conzelmann KK. Nonstructural proteins NS1 and NS2 of bovine respiratory syncytial virus block activation of interferon regulatory factor 3. J Virol. 2003;77:8661-8.

17. Jin $H$, Zhou $H$, Cheng $X$, et al. Recombinant respiratory syncytial viruses with deletions in the NS1, NS2, SH, and M2-2 genes are attenuated in vitro and in vivo. Virology. 2000;273:210-8.

18. Schlender J, Bossert B, Buchholz U, et al. Bovine respiratory syncytial virus nonstructural proteins NS1 and NS2 cooperatively antagonize alpha/beta interferon-induced antiviral response. J Virol. 2000;74:8234-42.

19. Jin $\mathrm{H}$, Zhou $\mathrm{H}$, Cheng $\mathrm{X}$, et al. Evaluation of recombinant respiratory syncytial virus gene deletion mutants in African green monkeys for their potential as live attenuated vaccine candidates. Vaccine. 2003;21:3647-52.

20. Valarcher JF, Furze J, Wyld S, et al. Role of alpha/beta interferons in the attenuation and immunogenicity of recombinant bovine respiratory syncytial viruses lacking NS proteins. J Virol. 2003;77:8426-39.

21. Spann KM, Tran KC, Chi B, et al. Suppression of the induction of alpha, beta, and lambda interferons by the NS1 and NS2 proteins of human respiratory syncytial virus in human epithelial cells and macrophages. J Virol. 2004;78:4363-269.

22. Heinze B, Frey S, Mordstein M, et al. Both nonstructural proteins NS1 and NS2 of pneumonia virus of mice are inhibitors of the interferon type I and type III responses in vivo. J Virol. 2011;85:4071-84.

23. Buchholz UJ, Ward JM, Lamirande EW, et al. Deletion of nonstructural proteins NS1 and NS2 from pneumonia virus of mice attenuates viral replication and reduces pulmonary cytokine expression and disease. J Virol. 2009:83:1969-80.

24. Dhar J, Barik S. Unique nonstructural proteins of pneumonia virus of mice (PVM) promote degradation of interferon (IFN) pathway components and IFN-stimulated gene proteins. Sci Rep. 2016;6:38139.
25. Spann KM, Tran KC, Collins PL. Effects of nonstructural proteins NS1 and NS2 of human respiratory syncytial virus on interferon regulatory factor 3 , NF-kappaB, and proinflammatory cytokines. J Virol. 2005;79:5353-62.

26. Lo MS, Brazas RM, Holtzman MJ. Respiratory syncytial virus nonstructural proteins NS1 and NS2 mediate inhibition of Stat2 expression and alpha/ beta interferon responsiveness. J Virol. 2005;79:9315-9.

27. Elliott J, Lynch OT, Suessmuth Y, et al. Respiratory syncytial virus NS1 protein degrades STAT2 by using the Elongin-Cullin E3 ligase. J Virol. 2007:81:3428-36.

28. Chatterjee S, Luthra P, Esaulova E, et al. Structural basis for human respiratory syncytial virus NS1-mediated modulation of host responses. Nat Microbiol. 2017;2:17101.

29. Rabbani MA, Ribaudo M, Guo JT, et al. Identification of interferonstimulated gene proteins that inhibit human parainfluenza virus type 3 . J Virol. 2016:90:11145-56.

30. Jiang $D, G u o ~ H, X u C$, et al. Identification of three interferon-inducible cellular enzymes that inhibit the replication of hepatitis $C$ virus. J Virol. 2008;82:1665-78.

31. Sievers F, Wilm A, Dineen D, et al. Fast, scalable generation of high-quality protein multiple sequence alignments using Clustal omega. Mol Syst Biol. 2011;7:539.

32. Whelan JN, Tran KC, van Rossum DB, Teng MN. Identification of respiratory syncytial virus nonstructural protein 2 residues essential for exploitation of the host ubiquitin system and inhibition of innate immune responses. J Virol. 2016;90:6453-63.

33. Ramachandran A, Horvath CM. Paramyxovirus disruption of interferon signal transduction: STATus report. J Interf Cytokine Res. 2009;29:531-57.

34. Wu W, Tran KC, Teng MN, Heesom KJ, Matthews DA, Barr JN, Hiscox JA. The interactome of the human respiratory syncytial virus NS1 protein highlights multiple effects on host cell biology. J Virol. 2012:86:7777-89.

35. Bitko V, Shulyayeva O, Mazumder B, Musiyenko A, Ramaswamy M, Look DC, Barik S. Nonstructural proteins of respiratory syncytial virus suppress premature apoptosis by an NF-kappaB-dependent, interferon-independent mechanism and facilitate virus growth. J Virol. 2007:81:1786-95.

36. Ling Z, Tran KC, Teng MN. Human respiratory syncytial virus nonstructural protein NS2 antagonizes the activation of beta interferon transcription by interacting with RIG-I. J Virol. 2009;83:3734-42.

37. Ren J, Liu T, Pang L, Li K, Garofalo RP, Casola A, Bao X. A novel mechanism for the inhibition of interferon regulatory factor-3-dependent gene expression by human respiratory syncytial virus NS1 protein. J Gen Virol. 2011:92:2153-9.

38. Fensterl V, Sen GC. Interferon-induced Ifit proteins: their role in viral pathogenesis. J Virol. 2015:89:2462-8

39. Raychoudhuri A, Shrivastava S, Steele R, et al. ISG56 and IFITM1 proteins inhibit hepatitis C virus replication. J Virol. 2011:85:12881-9.

40. Saikia P, Fensterl V, Sen GC. The inhibitory action of P56 on select functions of E1 mediates interferon's effect on human papillomavirus DNA replication. J Virol. 2010;84:13036-9.

41. Andrejeva J, Norsted $\mathrm{H}$, Habjan M, et al. ISG56/IFIT1 is primarily responsible for interferon-induced changes to patterns of parainfluenza virus type 5 transcription and protein synthesis. J Gen Virol. 2013:94:59-68.

42. Daffis S, Szretter KJ, Schriewer J, et al. 2'-O methylation of the viral mRNA cap evades host restriction by IFIT family members. Nature. 2010;468:452-6.

43. Szretter KJ, Daniels BP, Cho H, et al. 2'-O methylation of the viral mRNA cap by West Nile virus evades ifit1-dependent and -independent mechanisms of host restriction in vivo. PLoS Pathog. 2012;8:e1002698.

44. Habjan M, Hubel P, Lacerda L, et al. Sequestration by IFIT1 impairs translation of 2'O-unmethylated capped RNA. PLoS Pathog. 2013;9: e1003663.

45. Kimura $T$, Katoh $H$, Kayama $H$, et al. Ifit1 inhibits Japanese encephalitis virus replication through binding to 5' capped 2'-O unmethylated RNA. J Virol. 2013:87:9997-10003

46. Kumar P, Sweeney TR, Skabkin MA, et al. Inhibition of translation by IFIT family members is determined by their ability to interact selectively with the $5^{\prime}$-terminal regions of cap0-, cap1- and 5'ppp- mRNAs. Nucleic Acids Res. 2014:42:3228-45.

47. Barik S. The structure of the $5^{\prime}$ terminal cap of the respiratory syncytial virus mRNA. J Gen Virol. 1993:74:485-90.

48. Liuzzi M, Mason SW, Cartier M, et al. Inhibitors of respiratory syncytial virus replication target cotranscriptional mRNA guanylylation by viral RNA-dependent RNA polymerase. J Virol. 2005;79:13105-15. 
49. Pichlmair A, Lassnig C, Eberle CA, et al. IFIT1 is an antiviral protein that recognizes 5'-triphosphate RNA. Nat Immunol. 2011;12:624-30.

50. Oh SW, Onomoto K, Wakimoto $M$, et al. Leader-containing uncapped viral transcript activates RIG-I in antiviral stress granules. PLoS Pathog. 2016;12:e1005444

51. Barik S. What really rigs up RIG-I? J Innate Immun. 2016;8:429-36.

52. Jiang $D$, Weidner $J M$, Qing $M$, et al. Identification of five interferon-induced cellular proteins that inhibit West Nile virus and dengue virus infections. J Virol. 2010;84:8332-41.

53. Krzyzaniak MA, Zumstein MT, Gerez JA, et al. Host cell entry of respiratory syncytial virus involves macropinocytosis followed by proteolytic activation of the F protein. PLoS Pathog. 2013;9:e1003309.

54. Diamond MS, Farzan M. The broad-spectrum antiviral functions of IFIT and IFITM proteins. Nat Rev Immunol. 2013;13:46-57.

55. Mudhasani R, Tran JP, Retterer C, et al. IFITM-2 and IFITM-3 but not IFITM-1 restrict Rift Valley fever virus. J Virol. 2013;87:8451-64.

56. Wada T, Penninger JM. Mitogen-activated protein kinases in apoptosis regulation. Oncogene. 2004;23:2838-49.

57. Atreya PL, Kulkarni S. Respiratory syncytial virus strain A2 is resistant to the antiviral effects of type I interferons and human MxA. Virology. 1999;261:227-41.

58. Sun $L$, Wu J, Du F, Chen X, Chen ZJ. Cyclic GMP-AMP synthase is a cytosolic DNA sensor that activates the type I interferon pathway. Science. 2013;339:786-91

59. Majumdar T, Chattopadhyay S, Ozhegov E, Dhar J, Goswami R, Sen GC, Barik S. Induction of interferon-stimulated genes by IRF3 promotes replication of Toxoplasma gondii. PLoS Pathog. 2015;11:e1004779.

\section{Submit your next manuscript to BioMed Central} and we will help you at every step:

- We accept pre-submission inquiries

- Our selector tool helps you to find the most relevant journal

- We provide round the clock customer support

- Convenient online submission

- Thorough peer review

- Inclusion in PubMed and all major indexing services

- Maximum visibility for your research

Submit your manuscript at www.biomedcentral.com/submit

C) Biomed Central 